\title{
Estimation of Content Spread on NDN
}

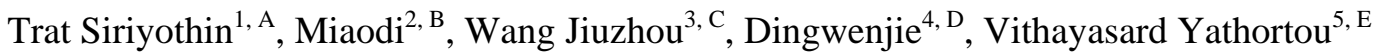 \\ School of Electronic Engineering, Tianjin University of Technology and Education,
}

Tianjin, China 300222

\begin{abstract}
In currently. IP architecture have more important in daily life. But because the IP architecture still has problems. When there are a large number of users Will cause data collisions and Security in data transfer and a lot of costs. Therefore, Named Data Networking or NDN is an interesting new architecture. By using the name as the center, Unlike IP architecture that will use host as the center. NDN has a unique name packet for each package. And forward to the router to find the route to the destination.[1] Naming the data rather than the location enables NDN routers to cache each packet and improve network bandwidth, the better. The spread of content will use a lot of computed bandwidth of NDN to distribute the content with the caching function. this paper will Explain the examination of the spread of content for Name Data Networking by running an implementation of NDN by using CCNx. NDN's content distribution tests have shown that content packet sizes have a greater impact on system workloads and poor $\mathrm{CCNx}$ decisions [2] will increase system workloads in the $\mathrm{CCNx}$ program. In addition, found That inefficient applications will cause unnecessary calculations to be decoded.

This article, in section1, Explain the experimental content of the initial distribution. section 2, shows the background for content distribution in NDN, for section 3 shows design and experimentation, section 4 describes experimental performance, and sections 5 and 6 show performance improvements and profiling results $\mathrm{CCNx}$.
\end{abstract}

Keywords-Named Data Networking (NDN), Content-Centric Network (CCNx), Content Spread, Performance Examination.

\section{INTRODUCTION}

The internet was created with the purpose of providing communication between hosts. the important aspects of content distribution are the host. Lots of requests for the same content must be transferred multiple times over the network from the server, which is clearly not effective.[3] Focusing on the content will have the two main NDN properties, namely 1. Packet with specific name Will be sent according to the search results of the packet name There are two types of packets in the NDN network: interesting packets and data packets. When users need some content will send interesting

packets to show requests the data packets are used to respond to interest packets with appropriate content.[4] 2. NDN routers can store a number of forwarded data packet caches. When the interest packet has arrived at the router. The router will have the content requested. Content, which will be sent back to the host immediately instead of forwarding the request to this server. [5] This can reduce a lot of bandwidth

in the NDN network and support better content distribution, Therefore, this test will measure the efficiency of the speed of packet content distribution in the active NDN and find a suitable solution to the current bandwidth problem.

\section{BACKGROUND}

This section 2 presents the content distribution systems, Named Data Networking and testbed.

a) NDN PRIME

From each node of the NDN network will run the daemon program. There is no difference between the client and the router server. But in the network, there are two types of packets which are : (1) Interest Packet and (2) Data Packet in which the packet names are arranged in a hierarchy for flexibility in the operation of the server router. [4]

In NDN's architecture, there are three main components. For forwarding [5] are: (1) Forwarding Information Base (FIB): Responsible for stores forwarding lists that send interest packets to the possible source of matching Data. Unlike IP architecture, that allows more than one list of exported faces (next-hop) for each name prefix. The FIB be populated manually and/or by The NDN routing protocol in the control plane. (2) Pending Interest Table. (PIT): Responsible for collecting the unsatisfied interest packets and received faces, so data packets can be returned to nodes interested in data. and (3) Content Store (CS): Responsible used for caching data.

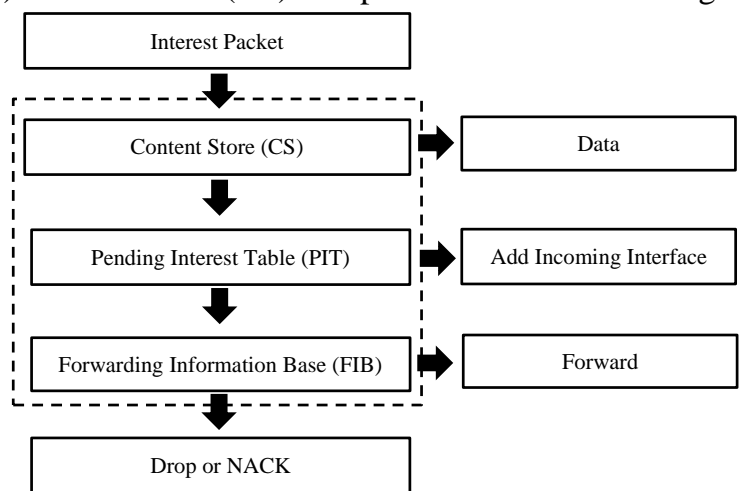

Figure1. forwarding plane of NDN

When attention arrives at the NDN router, the CS will be examined first for the corresponding data. If the CS can respond to the attention, the data packets will be sent back to the receiving face. Otherwise, it will be added to the PIT. If there is an item with the same name, a new face number will be added to the face list to be able to send a copy of the matching data packet on all the faces that the interest packet has arrived. and Eventually, if interest does not have a matching PIT item, it will forward the attention to the nexthop(s) according to the FIB. If there are many more hops in the FIB list, a module called "Forwarding strategies" will determine how to use multiple routes to pass attention on.[6] 


\section{b) Content distribution}

Normally, the distribution of NDN content affects many users. There are many methods, which are Client-Server method the server that stores content and clients can receive content directly from the server to reduce the workload of the server cache. [7] In the second method, a content distribution network (CND) is a network for displaying data and storing in many places. CDN will travel through the network. Gather the closest content with full use of the content delivery area for participants. And the last method is Peer-to-Peer (P2P). In this method it is divided into small sections and the host will download the content according to the group. [8] When the host has finished downloading some content Will continue to act as the content provider for that data the router is close to other preferred hosts, and P2P is still very popular in the network for distributing NDN content and is the easiest way for NDN networks.

\section{c) Implementation of prototypes}

The forward design of the NDN developed in PARC is a Content-Centric Networking Project (CCNx). The Important content of $\mathrm{CCNx}$ is the cond daemon.[9] It is a program intended for packets to be forwarding and caching. Generally, NDN does not use IP for real work. If having a real NDN network, IP networks can be used as overlays in the NDN network. In the CCNx project, there are three formats used in the CCNx network are : 1.Data Structures: Logical FIB and PIT use hash tables that provide Name Prefix Hash Table (NPHT), which indexes the Propagating Entries (PEs) and Forwarding Info Entries (FIE) There will be a position to store interest and forwarding information respectively. The Propagating Hash Table (PHT) is sent via the packet space that is shown in PEs and PHT, preventing replay in the network. Each content packet in the CS will receive a unique number. For daemon entry. Content Array (CA) is an array will store cached content packets. Straggler Hash Table (SHT) will collect some of the content packets to increase space efficiency. And there are two types of data structures: Content Hash Table (CHT) and Content Skip List (CSL) for making indicators that store content.
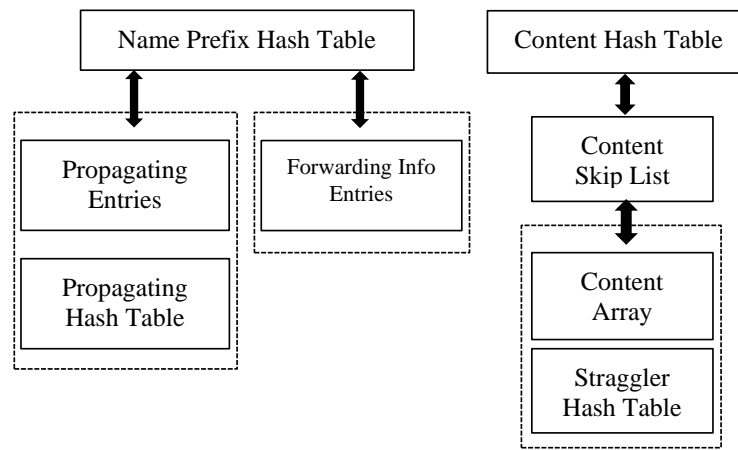

Figure 2. CCNx Forwarding Data Structures

CHT is a hash table with the name of the content packet. and The CSL will arrange a content-order lookup.[6] 2. Interesting packet processing: When the router receives the interest packet. will ask PHT first to make sure that no loops occur. CSL provides with search based on reliable queries and matched content. From CS, content will be pulled out, conducting a comprehensive matching examination. If the match is satisfactory Content packages will be sent to users who send this attention packet. If the matching is not satisfactory or there is no match, the daemon will find an interesting name on NPHT. The longest prefix match will be performed to find the appropriate FIE.[10] The interest packet will be sent to that host and will be added to PIT and PHT 3. Content packet processing: CHT will ask when the content packet arrives. To ensure that this packet is not stored in CS. If the packet is not repeated, it will be inserted into CSL and CS. After searching on CSL, NPHT will be asked the longest matching prefix to find all interest packets that can be made with this content packet. The content packet will be sent to that host.

\section{DESIGN}

To assess the content distribution in NDN has to systematically designed our tests and evaluations of the first results obtained. by selecting the metric to use for evaluating the performance of parameters the results obtained from the tests show the effect of the performance in the mentioned systems. and explain the technique for evaluation results and experimental designs $2^{\mathrm{k}}$

\section{a) System Explanation}

NDN has a distributed system consisting of three components: are NDN Clients, NDN Servers and NDN routers. These three components run the same ccnd daemon program for the corresponding host in the system. To be able to forward and caching packets. The client runs on the client-end software. While the server uses the server-end application running on ccnd.[11] Other applications do not run on the router. But will be forwarded in the content distribution, the content is stored on the server. The client will download the content by sending interest packets. interest packets Respond to application servers by sending content packets. follow the content packet will be cached in the content store of the NDN router.

\section{b) Indicators}

Examination of the spread of Content for NDN in the context of the NDN. Should choose indicators that show the quality of service the system generates. By using the necessary functions and provided by the content distribution system used on NDN, namely distributing content to end-users.

the ideal indicators are Content Download Time (CDoT) of destination host and Content Distribution Time (CDiT) of the system.[12] CDoT is the time between the client sending the first interest packet and receiving all content packets. CDiT is the time period that sends the first interest packet between clients or all clients that received that content.[13]

For evaluation, NDN router forwarding rate will be used as an evaluation measure. Can measure the NDN forwarding rate using a router based on NP-based. and when the CPU is saturated, the forward rate of the NDN router is set at the maximum throughput that the NDN router can accept for evaluation.

\section{c) Parameters}

There are many parameters in a computer network that can affect the performance of the NDN-based content distribution system. In this section, all parameters are described before the workload parameters. 


\begin{tabular}{|l|c|}
\multicolumn{1}{|c|}{ Table 1. Parameters } & Description \\
\hline Operating System & Ubuntu16.1 \\
\hline Transmission Protocol & TCP or UDP \\
\hline Number of Servers & 8 \\
\hline Number of Clients & 4 \\
\hline Number of Routers & 4 \\
\hline Server Application & 1 \\
\hline Client Application & 1 \\
\hline Network Topology & Tree \\
\hline Network Reliability & Configurable \\
\hline
\end{tabular}

System performance studies in Table 1 show software parameters. The CCNx can be configured and modified on each node. While the hardware parameters cannot be modified as easily as the software parameters.

Table 2. Workload Parameters

\begin{tabular}{|l|l|}
\hline \multicolumn{1}{|c|}{ Parameters } & Description \\
\hline Content Name Length & Configurable \\
\hline Content Packet Size & Configurable \\
\hline Content Diversity & Configurable \\
\hline
\end{tabular}

Since there is no NDN packet tracking, therefore, the design and use of simulated workloads for this evaluation study. Table 2 lists the workload parameters for this performance study.

Table 3. Factors to Study

\begin{tabular}{|l|l|}
\multicolumn{1}{c}{ Table 3. Factors to Study } \\
\begin{tabular}{|l|l|}
\hline \multicolumn{1}{|c|}{ Factors } & Description \\
\hline Content Store Capacity & $1 \mathrm{~K}, 10 \mathrm{~K}, 100 \mathrm{~K}$ \\
\hline Content Name Length & $2,4,8,16,32,64$ \\
\hline Content Packet Size & $64 \mathrm{~B}, 128 \mathrm{~B}, \ldots, 1 \mathrm{MB}$ \\
\hline Content Diversity & $1,2,4,8,16$ \\
\hline
\end{tabular}
\end{tabular}

Determinants are selected from the parameters specified in Table1 and Table 2. The main factors of the study listed in Table 3. NDN routers can change the capacity of the configured content store. Usually, CS that are larger can store more content and make it more likely to respond to requests that arrive.[14] The average time of a larger CS increases search and recovery time. Each NDN packet is assigned a unique name. And was assigned the path by name. Many elements of the NDN packet name, each consisting of a slash symbol.

NDN packet name length is determined as a component in the name by Long names increase search time and affect the workload of the system. [15] There is a hypothesis that every host request only one content. The amount of content that is varied is the amount of different content requested by the host.

The parameters are modified using the program. Ccncatchunks 2 to download the client Which will respond to random data interests Which consists of server number 4 and the client set to 8 . This number is set to ensure that the NDN router's CPU can be saturated. In order to be able to measure the maximum workload of the system, choose TCP as the transmission protocol for this assessment. Figure 3 shows the network topology It can be seen that there are 4 NDN servers, namely Server1 to Server4. That are connected to 8 Host NDN is Host1 through Host7 through. A router NDN NP is R1 through R4. Used for network connections and measures the amount of data transmitted by the system.

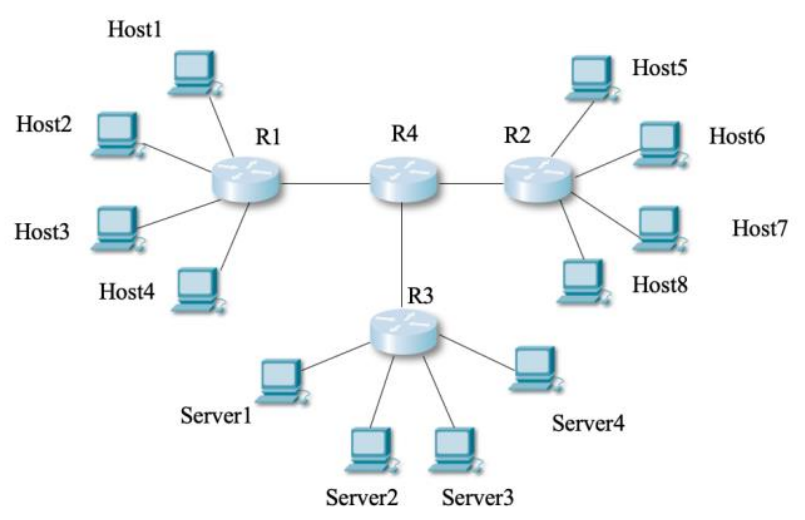

Figure 3. Network Topology

\section{d) Evaluation}

The program used in the experiment have been developed and study the measurement of the system using the network topology created using CCNx [16], as have seen in Figure 3, with script development. Some to control the hosts participating in the test the output of the NDN router is measured by a router using NP in Network. The measured data is displayed directly in the Terminal.

\section{e) Experimental Design}

Since there are 4 factors in the study Therefore, use the $2^{\mathrm{k}}$ factor design for the design of the experiment, where $\mathrm{k}=4$. This gives the experimental result $2^{4}=16$. The list of factors and levels of each factor in Table 4.

Table 4. Factor Symbol and Levels

\begin{tabular}{|c|l|c|c|}
\hline Symbol & Factor & Level -1 & Level +1 \\
\hline A & Content Store Capacity & $10 \mathrm{~K}$ & $50 \mathrm{~K}$ \\
\hline B & Content Name Length & 4 & 16 \\
\hline C & Content Packet Size & $64 \mathrm{kB}$ & $1 \mathrm{MB}$ \\
\hline D & Content Diversity & 1 & 8 \\
\hline
\end{tabular}

\section{EFFICIENCY}

This section provides a description of the performance of the content distribution system on the NDN. Results are presented and explained in this section. By using data analysis techniques to identify key factors affecting distribution efficiency.

Table 5. Measured System Throughput

\begin{tabular}{|c|c|c|c|c|c|c|}
\hline$\#$ & A & B & C & D & $\begin{array}{c}\text { Bandwidth } \\
\text { Mbps }\end{array}$ & $\begin{array}{c}\text { Package } \\
\text { Mbps }\end{array}$ \\
\hline $\mathbf{1}$ & -1 & -1 & -1 & -1 & 7.2 & 41.72 \\
\hline $\mathbf{2}$ & 1 & -1 & -1 & -1 & 7.69 & 45 \\
\hline $\mathbf{3}$ & -1 & 1 & -1 & -1 & 8.23 & 48 \\
\hline $\mathbf{4}$ & 1 & 1 & -1 & -1 & 8.65 & 49.19 \\
\hline $\mathbf{5}$ & -1 & -1 & 1 & -1 & 9.83 & 55.78 \\
\hline $\mathbf{6}$ & 1 & -1 & 1 & -1 & 10.36 & 58 \\
\hline $\mathbf{7}$ & -1 & 1 & 1 & -1 & 11 & 59 \\
\hline $\mathbf{8}$ & 1 & 1 & 1 & -1 & 13.47 & 64.48 \\
\hline $\mathbf{9}$ & -1 & -1 & -1 & 1 & 14.46 & 67.76 \\
\hline $\mathbf{1 0}$ & 1 & -1 & -1 & 1 & 16.62 & 70 \\
\hline $\mathbf{1 1}$ & -1 & 1 & -1 & 1 & 20 & 75.4 \\
\hline $\mathbf{1 2}$ & 1 & 1 & -1 & 1 & 22.6 & 78.7 \\
\hline $\mathbf{1 3}$ & -1 & -1 & 1 & 1 & 23.7 & 89 \\
\hline $\mathbf{1 4}$ & 1 & -1 & 1 & 1 & 25.27 & 99.45 \\
\hline $\mathbf{1 5}$ & -1 & 1 & 1 & 1 & 30 & 103 \\
\hline $\mathbf{1 6}$ & 1 & 1 & 1 & 1 & 31 & 121 \\
\hline
\end{tabular}




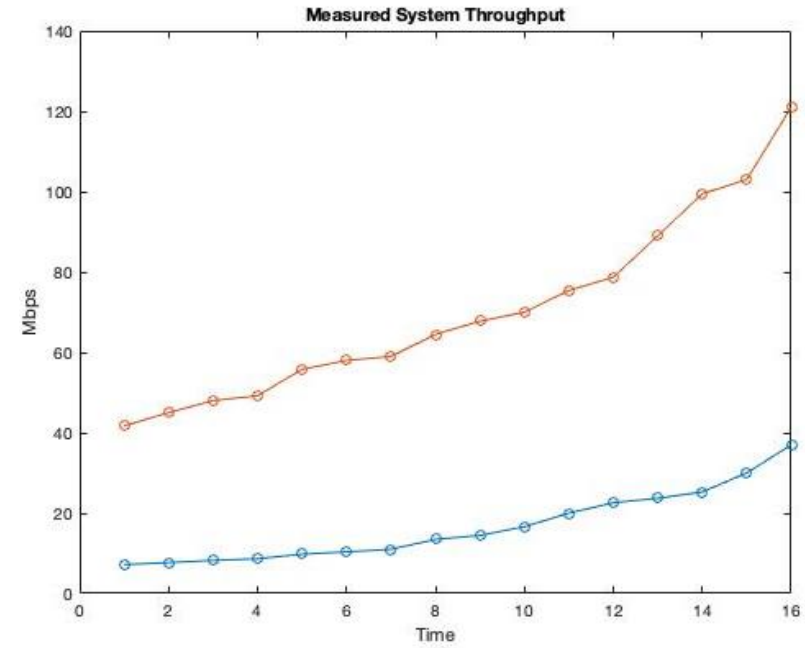

Figure 4 Measured System Throughput

a) Operating Results

After the experiment design in Table 5 and Figure 4. There are 16 experiments. The amount of incoming traffic going into the NDN router and the outbound traffic quantity from the router. will be measured using the traffic test tools available on NP-routers. Bandwidth usage is shown in Table. And the value of the workload Can be read directly from SSH. The total workload of the 16 measured systems is in Table 5 . With a minimum bandwidth load of 7.2 Mbps and a package starting at $41.72 \mathrm{Mbps}$ in 16 tests, the package volume from the 12th Will increase dramatically, the workload bandwidth will increase step by step, respectively Which the bandwidth and packages are still very far apart.

Table 6. Top 5 Contributors to the Variation
\begin{tabular}{|c|c|}
\hline Symbol & Var (\%) \\
\hline C & 51 \\
\hline B & 22 \\
\hline BC & 9.5 \\
\hline D & 9 \\
\hline CD & 5.6 \\
\hline
\end{tabular}

\section{b) Data analysis}

Assessing the factors that affect the system and the highest efficiency from the analysis of the data specified in Table 5 or Figure 5 , the top 5 factors in terms of participation in the changes specified in Table 6 . from the data in Table 6 , factors $\mathrm{C}$ are Content size and the most important factor affecting the workload of the system. Factor B is the length of the content title, the second priority of the $\mathrm{BC}$ factor table is the intersection of two factors. Wash is the third factor. Followed by the $\mathrm{D}$ factor is the content diversity, the $\mathrm{CD}$ factor is the intersection of the two factors $\mathrm{C}$ and $\mathrm{D}$. In addition, Factor A, the storage size of the content is insignificant in terms of impact on system workloads.

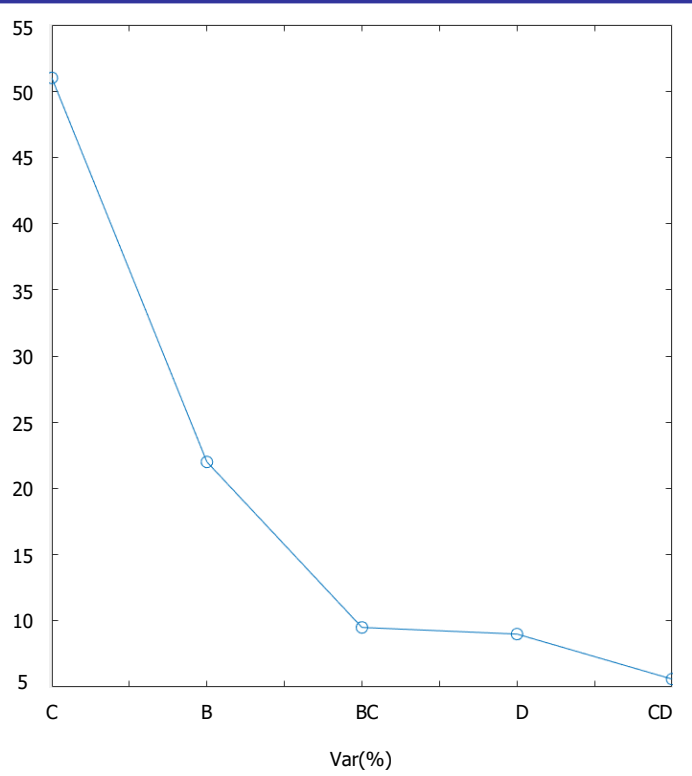

Figure 5. Contributors to the Variation

\section{IMPROVING PERFORMANCE}

The tests in the previous section had the highest workload used. The speed that can be achieved is 7.2 Mbps and the maximum is $31 \mathrm{Mbps}$, which results in an increase in volume. But it takes longer for the data to be transmitted more efficiently. Line speed compared to current speed to illustrate that NDN has the ability to provide content distribution functions and other methods of content distribution, which are important in improving and increasing system performance.

This section explains how to increase the forwarding rate. by changing the source code can increase the number of forwarding nodes by up to double to improve forwarding, there are two basic methods: 1) Designing or using data structures for a more effective search for packet names. 2) Rent or buy advanced server hardware to find an efficient route. Today, server hardware has the ability and capability to manage a system on an NDN network. It can find the longest prefix which can increase speed. In addition, the use of nodes for forwarding NDNs on the network processor can improve performance.

Table 7. Top 10 Time-Consuming Functions

\begin{tabular}{|l|c|}
\hline \multicolumn{1}{|c|}{ Function Name } & Percentage (\%) \\
\hline ccn_skeleton_decode & 47.34 \\
\hline ccn_buf_advance & 7.58 \\
\hline ccn_compare_names & 6.1 \\
\hline hashtb_hash & 5.87 \\
\hline ccn_buf_match_dtag & 3.55 \\
\hline ccn_parse_Signature & 3.11 \\
\hline ccn_buf_match_blob & 2.01 \\
\hline content_skiplist_findbefore & 1.48 \\
\hline hashtb_seek & 1.36 \\
\hline content_from_accession & 1.21 \\
\hline The Rest & 20.39 \\
\hline Sum & 100 \\
\hline
\end{tabular}


CCNx is a tool that has the potential to improve the efficiency of the application to suit the current application. the top 10 long-time functions. All functions are from ccn.h and coding.h, which show the CPU processing time of decoding in Table 7 and Figure 6-4 shows the italic function as a function. Related to decoding packets, as in Table 7, more than half of the time it takes to decipher a particular packet name. ccn_skeleton_decode That takes as much as 47.34 percent.

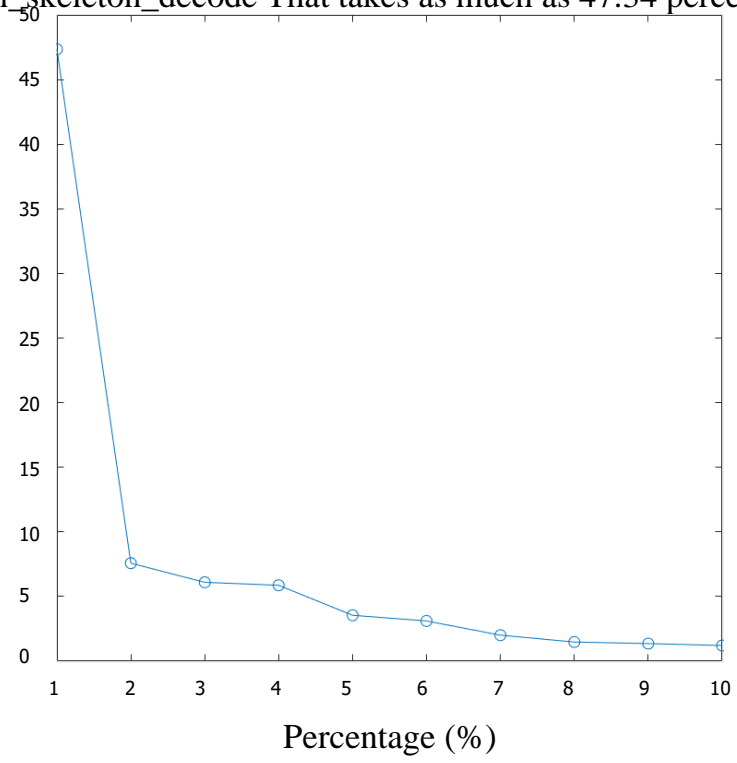

Figure 6. 10 Time-Consuming Functions

Which takes an unusually long time in general network programs. What causes the problem is the content packets to be stored in the Content Store (CS) are encoded. When inquiring about skipping content in the $\log (\mathrm{n})$ packet, it must be decoded immediately. Where $\mathrm{n}$ is defined as the number of items stored in the Content Skip List (CSL). Which will take a very long time When making program changes. In which the name of the content packet that is decoded Will be stored in the daemon. In this way, there will be no special packet decoding when CSL is asked each time.

\section{CONCLUTION}

In this article, assess the effectiveness of the content distribution system for the named data network. Which will check the workload of NDN. The experiment will show that the workload of CCNx in the operating system Still far from current demand and experiments show that the key factor is the size of the content packet may have the greatest impact on performance and the number of storage name prefixes that will affect. Forwarding if it is too small in comparison to the current application.

\section{REFERENCE}

[1] Saxena, Divya, Vaskar Raychoudhury, Neeraj Suri, Christian Becker, and Jiannong Cao. "Named data networking: a survey." Computer Science Review 19 (2016): 15-55.

[2] Shailendra, Samar, Senthilmurugan Sengottuvelan, Hemant Kumar Rath, Bighnaraj Panigrahi, and Anantha Simha. "Performance evaluation of caching policies in ndn-an icn architecture." In 2016 IEEE Region 10 Conference (TENCON), pp. 1117-1121. IEEE, 2016.

[3] Greenberg, Albert, Parantap Lahiri, David A. Maltz, Parveen K. Patel, Sudipta Sengupta, Navendu Jain, and Changhoon Kim. "Agile data center network architecture." U.S. Patent 9,497,039 issued November 15, 2016

[4] Hassan, Suhaidi, Walid Elbreiki, Mohamed Firdhous, and Adib M. Monzer Habbal. "End-to-end networks vs named data network: A critical evaluation." Jurnal Teknologi 72, no. 5 (2015): 71-76.

[5] Aubry, Elian, Thomas Silverston, and Isabelle Chrisment. "Implementation and evaluation of a controller-based forwarding scheme for NDN." In 2017 IEEE 31st International Conference on Advanced Information Networking and Applications (AINA), pp. 144151. IEEE, 2017.

[6] Lehman, Vince, AKM Mahmudul Hoque, Yingdi Yu, Lan Wang, Beichuan Zhang, and Lixia Zhang. "A secure link state routing protocol for NDN." Tech. Rep. NDN-0037 (2016).

[7] Marchal, Xavier, Thibault Cholez, and Olivier Festor. "Server-side performance evaluation of NDN." In Proceedings of the $3 r d$ ACM Conference on Information-Centric Networking, pp. 148-153. ACM, 2016

[8] Detti, Andrea, Bruno Ricci, and Nicola Blefari-Melazzi. "Mobile peerto-peer video streaming over information-centric networks." Computer Networks 81 (2015): 272-288.

[9] Garcia-Luna-Aceves, J. J., and Maziar Mirzazad Barijough. "Contentcentric networking using anonymous datagrams." In 2016 IFIP Networking Conference (IFIP Networking) and Workshops, pp. 171-179. IEEE, 2016.

[10] Majed, Al-qutwani, Xingwei Wang, and Bo Yi. "Name Lookup in Named Data Networking: A Review." Information 10, no. 3 (2019): 85

[11] Ariefianto, W. Tody, and Nana Rachmana Syambas. "Routing in NDN network: A survey and future perspectives." In 2017 11th International Conference on Telecommunication Systems Services and Applications (TSSA), pp. 1-6. IEEE, 2017.

[12] Rehman, Rana Asif, Tran Dinh Hieu, Hong-Min Bae, Sung-Hoon Mah, and Byung-Seo Kim. "Robust and efficient multipath Interest forwarding for NDN-based MANETs." In 2016 9th IFIP Wireless and Mobile Networking Conference (WMNC), pp. 187-192. IEEE, 2016.

[13] Nam, SunWook, Dohyung Kim, and Ikjun Yeom. "Content verification in Named Data Networking." In 2015 International Conference on Information Networking (ICOIN), pp. 414-415. IEEE, 2015.

[14] Hemanathan, V., and N. Anusha. "Role based content access control in NDN." J. Innov. Technol. Educ 2, no. 1 (2015): 65-73.

[15] Feng, Shuaibo, Mingchuan Zhang, Ruijuan Zheng, and Qingtao Wu. "A fast name lookup method in NDN based on hash coding." In 2015 3rd International Conference on Mechatronics and Industrial Informatics (ICMII 2015). Atlantis Press, 2015.

[16] Lailari, Ze'ev, Hila Ben Abraham, Ben Aronberg, Jackie Hudepohl, Haowei Yuan, John DeHart, Jyoti Parwatikar, and Patrick Crowley. "Experiments with the Emulated NDN Testbed in ONL." In Proceedings of the 2nd ACM Conference on Information-Centric Networking, pp. 219-220. ACM, 2015. 